\title{
SNR and BER Performance Analysis of MRC and EGC Receivers over Rayleigh Fading Channel
}

\author{
Navdeep Kaur \\ Department of Electronics Technology, \\ Guru Nanak Dev University, Punjab, India
}

\begin{abstract}
In this paper, the performance of an uncoded Single Input Multiple Output (SIMO) scheme is analyzed under Rayleigh fading channel using Maximum Ratio Combining (MRC) and Equal Gain Combining (EGC) methods. The analysis of these combining methods is done on the basis of two major factors, Signal to Noise Ratio (SNR) and Bit Error Rate (BER) performance using QAM and PSK modulation techniques. With increase in number of antenna on receiver side, SNR gets maximized and BER gets minimized. It is analyzed that MRC with QAM modulation has shown better evaluation results than EGC.
\end{abstract}

\section{Keywords}

Rayleigh fading, Bit Error Rate (BER), Maximum Ratio Combining (MRC), Equal Gain Combining (EGC), SingleInput-Multiple-Output (SIMO), MIMO systems.

\section{INTRODUCTION}

In a multipath environment, diversity techniques are used to enhance the capacity of wireless communication system. A technique named Multiple Input Multiple Output (MIMO) is very popular in modern high-speed wireless system due to enhancing capabilities. In this technique, multiple antennas are used at the transmitter and receiver to increase the capacity of the system. Another version of MIMO is Single Input Multiple Output (SIMO) occurs when a single antenna is on transmitter side and multiple antennas are on receiver side. This is also named as receive diversity. Since a receiver system receives signals from a number of independent sources, hence the diversity techniques enable it to combat the consequences of fading [1]. To achieve diversity gain, two techniques are broadly used named Maximum Ratio Combining (MRC) and Equal Gain Combining (EGC). MRC is a commonly used combining method to ameliorate the performance of noise limited communication systems. In this technique, the signals received from all diversity branches are weighted and combined to maximize Signal to Noise (SNR) at the combiner output. EGC is similar to MRC having an exception to overlap the weighing circuits [2]. The performance improvement is little bit lower in EGC than MRC because there is a chance to combine the noise signals with the noise-free high quality signals. The procedure of EGC is combining the individual signal branch coherently but it combines some noise components non- coherently. This paper presents the analysis of maximum ratio and equal gain combining for wireless communications, where multiple antennas are used for reception. Simulation results include BER and SNR comparison for different modulation schemes for MRC and EGC. Theoretical analysis is verified by simulation in Matlab.

\section{SYSTEM MODEL}

In order to meet the demand for high-speed data rates of wireless system, multiple antenna (MA) systems have become an indispensable part. When multiple antenna are only available at the receiver, combining schemes such as EGC, and MRC have been used to get diversity merits from the corresponding SIMO wireless channel [2]. In wireless communications, diversity is one of the most powerful means (best tradeoff between performance and cost) to combat multipath fading. It increases the steepness of the slope of the error probability versus SNR so that the SNR needed for a certain target error probability is greatly reduced, without increasing the transmitted signal power or deceasing system bandwidth efficiency [3].

There are $\mathrm{N}$ multiple receive antennas for one transmit antenna. The channel experienced by each receive antenna is varying in time randomly. For the $\mathrm{N}^{\text {th }}$ receive antenna, each transmitted symbol gets multiplied by a randomly varying complex number $h_{i}$, As the channel under consideration is Rayleigh fading channel, the real and imaginary parts of $u_{h_{i}}=0$ are Gaussian distributed having mean is zero and variance

$$
\sigma_{h_{i}}^{2}=\frac{1}{2} \text {. }
$$

The channel experienced by each receive antenna is independent from the channel experienced by other receive antennas. On each receive antenna, the noise $n$ has the Gaussian probability density function with

$$
\begin{aligned}
p(n)= & \frac{1}{\sqrt{2 \pi \sigma^{2}}} \\
& \text { with } \mu=0 \text { and } \sigma^{2}=\frac{N_{o}}{2} .
\end{aligned}
$$

The noise on each receive antenna is independent from the noise on the other receive antennas. At each receive antenna, the channel hi, known at the receiver. In the presence of channel hi, the instantaneous bit energy to noise ratio at $i^{\text {th }}$ receive antenna is

$$
\gamma_{i}=\frac{\left|h_{i}\right|^{2} E_{b}}{N_{o}}
$$

for rotational convenience.

\subsection{Maximum Ratio Combining (Mrc)}

In the MRC combining technique, summing circuits, weighting and co-phasing are required. The signals are co-phased and weighted before summing or combining from distinct diversity branches [2]. For maximizing the combined Carrier to Noise Ratio (CNR), the weights have to be chosen as proportional to the respective signals level [4]. The applied weighting to the each diversity branch has to be adjusted corresponding to the value of SNR. 


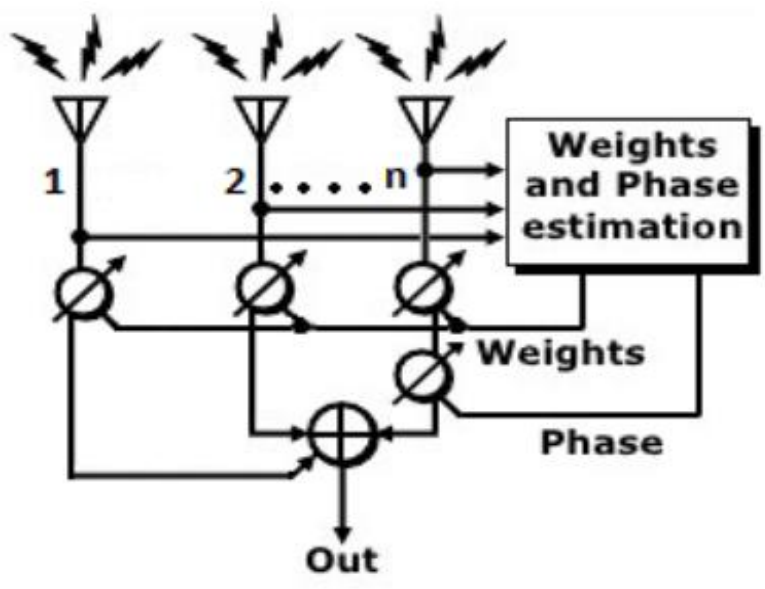

Fig 1: Maximum Ratio Combining

This is a very useful combining process. To contend against channel fading, this is the best combining process as it achieves the best performance improvement comparing to other methods. The MRC is most commonly used combining method to improve performance in a noise limited communication systems where the AWGN and the fading are independent amongst the diversity branches. It is also known as predetection and ratio-squared combining [5]. MRC is the most ideal diversity combining technique.

On the $i^{t h}$ receiver antenna, the received signal is,

$$
y_{i}=h_{i} x+n_{i}
$$

$y_{i}=$ received symbol on the $i^{\text {th }}$ receive antenna

$h_{i}=$ channel on the $i^{t h}$ receive antenna

$\mathrm{x}=$ transmitted symbol

$n_{i}=$ noise on $i^{t h}$ receive antenna

The equalized symbol is,

$\widehat{x}=\frac{h^{H} y}{h^{H} h}=\frac{h^{H} h x}{h}+\frac{h^{H} n}{h^{H} h}=x+\frac{h^{H} n}{h^{H} h}$

where $h^{H} h=\sum_{i=1}^{N}\left|h_{i}\right|^{2}$,

sum of the channel powers across all the receive antennas.

\subsubsection{Effective signal to noise ratio $\left(E_{b} / N_{o}\right)$ with} $M R C$

The instantaneous bit energy to noise ratio, in the presence of channel $h_{i}$ at $i^{\text {th }}$ receive antenna is

$$
\gamma_{i}=\frac{\left|h_{i}\right|^{2} E_{b}}{N_{o}}
$$

Equalizing channel with $h^{H}$, with the $\mathrm{N}$ receive antenna case, the effective bit energy is

(5)

$$
\gamma_{i}=\sum_{i=1}^{N} \frac{\left|h_{i}\right|^{2} E_{b}}{N_{o}}
$$

Effective bit energy to noise ratio in an $\mathrm{N}$ receive antenna case is $\mathrm{N}$ times the bit energy to noise ratio for single antenna [5].

\subsubsection{Bit Error Rate with $M R C$}

If $h_{i}$ is a Rayleigh distributed random variable then $h_{i}^{2}$ is a chisquared random variable with two degrees of freedom. The pdf of $\gamma$ is

$$
p\left(\gamma_{i}\right)=\frac{1}{E_{b} / N_{o}} e^{\frac{-\gamma_{i}}{E_{b} / N_{o}}}
$$

As the effective bit energy to noise ratio $\gamma$ is the sum of $\mathrm{N}$ such random variables, the pdf of $\gamma$ is a chi-squared random variable with $2 \mathrm{~N}$ degrees of freedom. The pdf of $\gamma$ is

$$
p(\gamma)=\frac{1}{(N-1) !\left(E_{b} / N_{o}\right)^{N}} \gamma^{N-1} e^{\frac{-\gamma}{\left(E_{b} / N_{o}\right)}} \quad ; \quad \gamma \geq 0
$$

BER figuring in AWGN, the effective bit energy to noise ratio with MRC is $\gamma$, the total bit error rate is the integral of the conditional BER integrated over all possible values of $\gamma[6]$.

$$
\begin{gathered}
P_{e}=\int_{0}^{\infty} \frac{1}{2} \operatorname{erfc}(\sqrt{\gamma}) p(\gamma) \mathrm{d} \gamma \\
=\int_{0}^{\infty} \frac{1}{2} \operatorname{erfc}(\sqrt{\gamma}) \frac{1}{(N-1) !\left(E_{b} / N_{o}\right)^{N}} \gamma^{N-1} e^{\frac{-\gamma}{\left(E_{b} / N_{o}\right)}} d_{\gamma}
\end{gathered}
$$

\subsection{Equal Gain Combining (EGC)}

The EGC resembles to MRC with an only exception to omit the weighting circuits. The performance improvement is lower in EGC than MRC only up to small extent because there is a chance to put together the signals with interference and noise, with the signals in high quality, which are interference and noise free. The individual signal branches are coherently combined but some noise components are non-coherently combine in EGC's normal procedure [2].

MRC is the most ideal diversity combining but the system requires very expensive design to adjust the gain in each branch at receiver circuit. It necessitates an appropriate tracking for the complex fading, which is practically very difficult to achieve. However, a simple phase lock summing circuit is used, due to this, it is very easy to implement an equal gain combining.

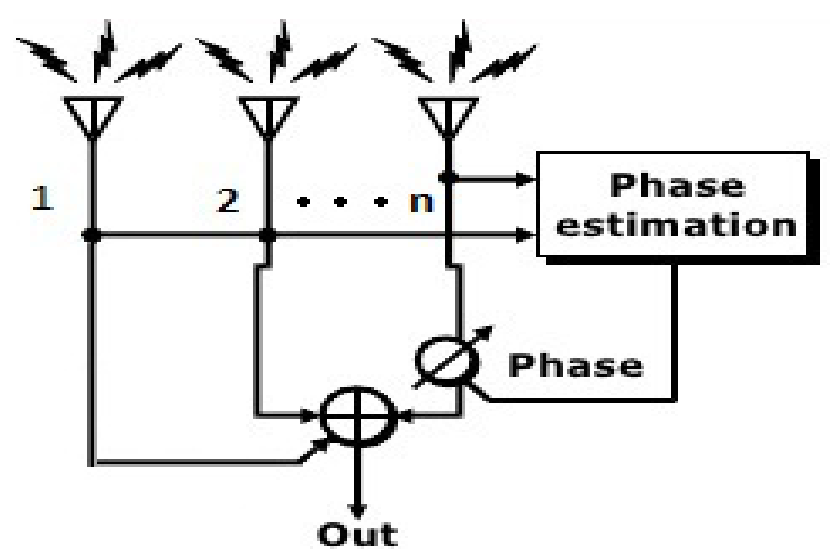

Fig 2: Equal Gain Combining

In the reception of diversity with coherent modulation EGC can be employed. The diversity branches are combined here with equal weights but conjugate phase and the envelope gains of diversity channels are neglected in EGC. The overall arrangement of EGC is as following, since there is no envelope gain estimation of the channel [7]. 
On the $i^{t h}$ receive antenna, equalization is performed at the receiver by dividing the received symbol $y_{i}$ by the known phase of $h_{i}$. The channel $h_{i}$ is represented in polar form as $\left|h_{i}\right| e^{j \theta_{i}}$. The decoded symbol in sum of the phase compensated channel from all the receive antennas.

$$
\begin{gathered}
\hat{y}=\sum_{i} \frac{y_{i}}{e^{j \theta_{i}}} \\
=\sum_{i} \frac{\left|h_{i}\right| e^{j \theta_{i}} x+n_{i}}{e^{j \theta_{i}}}
\end{gathered}
$$

$=\sum_{i}\left|h_{i}\right| x+\tilde{n}_{i}$

where $\tilde{n}_{i}=\frac{n_{i}}{e^{j \theta_{i}}} \quad$ is the additive noise scaled by the phase of the channel coefficient.

For PSK modulation schemes, the equalization by the phase of the channel coefficients is sufficient. However, for QAM modulation scheme case, compensate for the amplitude is also necessary when equalizing.

\subsubsection{Effective signal to noise ratio $\left(E_{b} / N_{o}\right)$ with EGC}

The instantaneous bit energy to noise ratio, in the presence of channel $h_{i}$ at $i^{\text {th }}$ receive antenna is

$\gamma_{i}=\frac{\left|h_{i}\right|^{2} E_{b}}{N_{o}}$. (10)

The effective Eb/No with equal gain combining is the channel power gathered from all receive antennas, which is the first term is chi-square random variable with $2 N$ degrees of freedom having mean value of $2 N \sigma_{h_{i}}^{2}$. Therefore, the first term becomes

$\sum_{i=1}^{N} h_{i}{ }^{2}=N$.

The second term is a product of two Rayleigh random variables. The mean of Rayleigh random variable with variance $\sigma_{h_{i}}^{2}$ is $\sigma_{h_{i}} \sqrt{\frac{\pi}{2}}$.

\subsubsection{Bit Error Rate (BER) with EGC}

The performance of an EGC receiver when phase shift keying or quadrature amplitude modulation is transmitted over a multipath channel with $\mathrm{N}$ paths for $\mathrm{N}$ receives antenna on the multipath flat fading amplitudes BER of an EGC receiver is given by [4]

$$
\begin{array}{r}
P_{e}(E)=Q\left(\sqrt{2 g \gamma_{e g c}}\right) \\
=Q\left(\sqrt{\frac{2 g E_{b}}{\sum_{i=1}^{N} N_{i}}\left(\sum_{i=1}^{N} a_{i}\right)^{2}}\right)
\end{array}
$$

where $\mathrm{E}_{\mathrm{b}} / \mathrm{N}_{\mathrm{o}}$ is the bit SNR on the AWGN channel, $a_{i}$ is fading amplitude after combining [6], $\mathrm{g}$ is a modulation dependent parameter such that $\mathrm{g}=1$ for BPSK and $\mathrm{Q}($.) is the Gaussian Qfunction defined by

(12)

$$
Q(x)=\frac{1}{\sqrt{2 \pi}} \int_{x}^{\infty} e^{-t^{2} / 2} d t
$$

The average BER $P_{e}(E)$ is obtained by averaging over the joint $\mathrm{PDF}$ of the channel fading amplitudes after combining is

$\int_{0}^{\infty} Q\left(\sqrt{\frac{2 g E_{b}}{\sum_{i=1}^{N} N_{i}}\left(\sum_{i=1}^{N} a_{i}\right)^{2}}\right)$

\section{CHANNELS}

For wireless communication, signal from transmitter is travel through space or air as a transmission media. The received signal at receiver is not only coming directly from the transmitter, but the combination of reflected, diffracted, and scattered copies of the transmitted signal, because radio propagation is not as smooth as in wire transmission [3].

\subsection{AWGN Channel}

Additive White Gaussian Noise (AWGN) channel is universal channel model for analyzing all modulation schemes. In this channel just add white Gaussian noise to signal passing through it, as it is known that it has uniform power across the frequency band for the overall information system [3]. It is used in information theory to mimic the effect of many random processes that occur in nature. It has a normal distribution in the time domain with an average time domain value of zero as only distortion is introduced by AWGN. The received signal is simplified to

$$
\mathrm{r}(\mathrm{t})=\mathrm{x}(\mathrm{t})+\mathrm{n}(\mathrm{t})
$$

where $n(t)$ is additive white Gaussian noise. The whiteness of $\mathrm{n}(\mathrm{t})$ implies that it is stationary random process with flat power spectral density (PSD) for all frequencies. So it is conventional to assume its PSD as follows

$$
N(f)=N o / 2,-\infty \leq f \leq \infty
$$

This implies that a white process has infinite power.

\subsection{RAYLEIGH Fading Channel}

In a wireless communication system, variations of the signal amplitude results in multipath fading because of the addition of signals arriving with different phases [8]. This received signal phase difference is caused due to the fact that signals have traveled different distances by traveling along different paths of a propagation environment of wireless media. Because the phases of the arriving paths are changing rapidly, amplitude and phase of the received signal undergoes rapid fluctuations that are often modeled as a random variable with a particular distribution [9]. The most commonly used distribution for multipath fast fading is the Rayleigh distribution, whose probability density function is given by

$$
f_{\text {ray }}=\frac{r}{\sigma^{2}} e^{-r^{2} / \sigma^{2}}, r \geq 0
$$

The random variable corresponding to the signal amplitude is $r$ and $\sigma 2$ is the variance. So Rayleigh flat fading channel is commonly used to describe multipath fading channels that affect the performance of a wireless communication network when there are no Line-Of-Sight (LOS) exists between transmitter and receiver [10].

\section{SIMULATIONS AND RESULTS}

The simulations in Matlab are presented to analyze Single Input Multiple Output (SIMO) scheme under Rayleigh fading channel combined using Maximal Ratio Combining (MRC) and Equal Gain Combining (EGC). The signal to noise (SNR) comparison using16-QAM and 16-PSK modulations derived for these two combining techniques for 20 numbers of receiver antennas. Then the bit error rate performance of MRC and EGC for two antennas using different modulation techniques is simulated and also the bit error rate for 16-PSK and 16-QAM for single receiver is analyzed. 


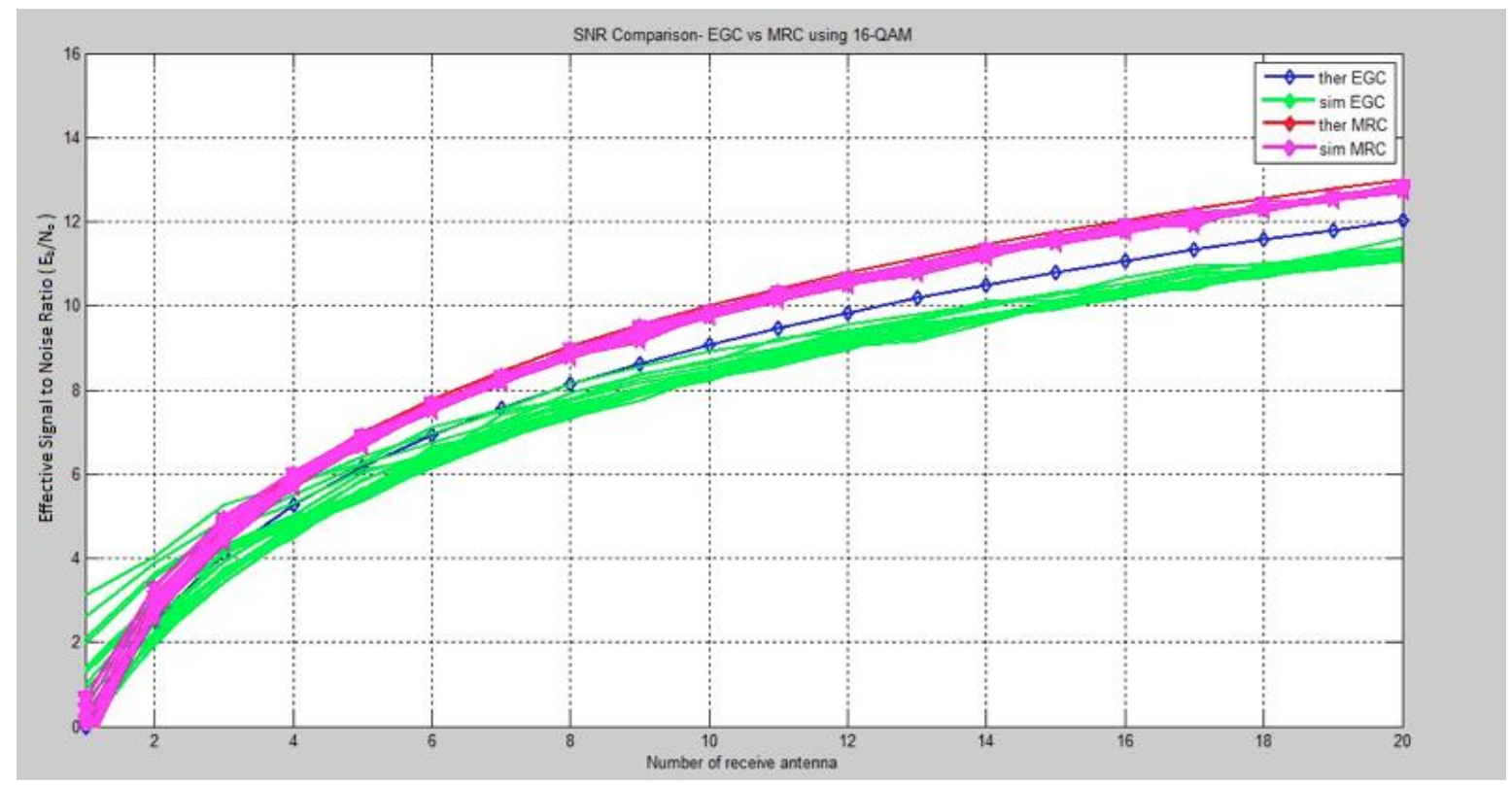

Fig 3: Effective $\operatorname{SNR}\left(\mathrm{E}_{\mathrm{b}} / \mathrm{N}_{\mathrm{o}}\right)$ comparison of EGC and MRC using 16-QAM modulation technique

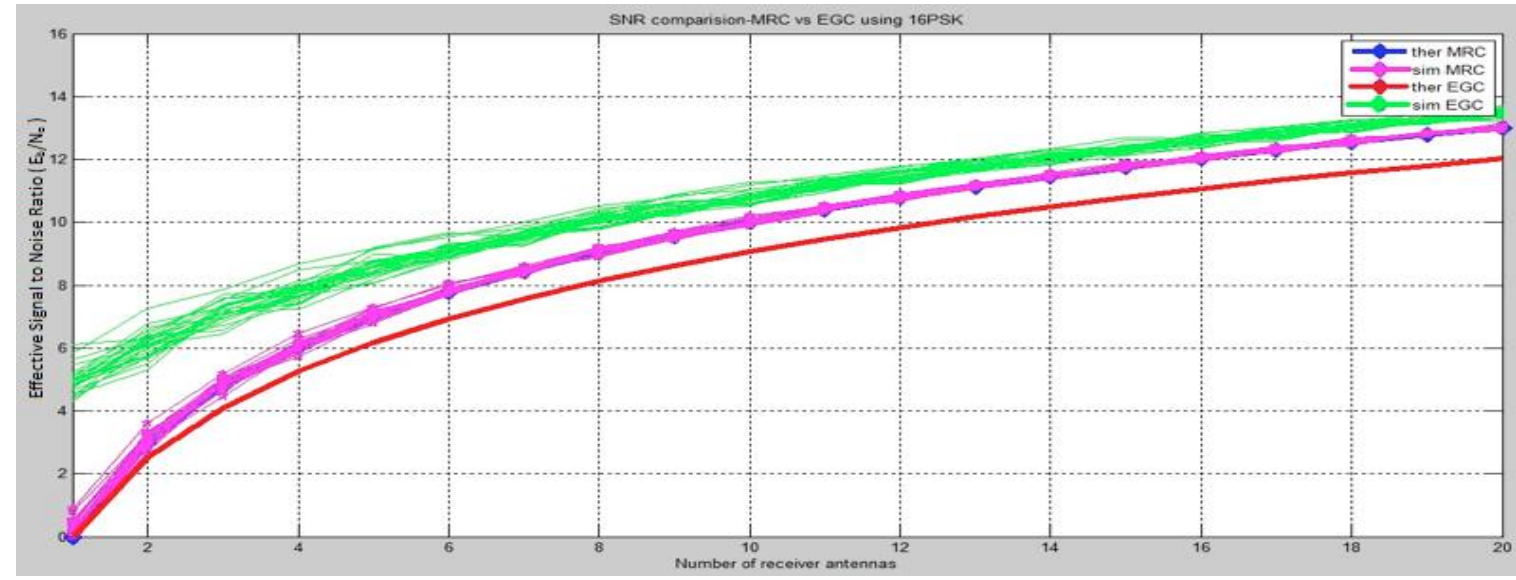

Fig 4: Effective SNR $\left(E_{b} / N_{o}\right)$ comparison of EGC and MRC using 16-PSK modulation technique

When the value of effective SNR (Eb/No) with number of different receive antennas is calculated, figure 3 and figure 4 shows that maximal ratio combining technique gives the better result as compare to the equal gain combining technique. Theoretical results are nearly equal to the simulated results in MRC for 16-PSK and 16-QAM modulation technique than EGC. However, 16-QAM technique shows better results than 16-PSK technique for same number of antennas in both MRC and EGC combining methods of diversity in Rayleigh fading channel.

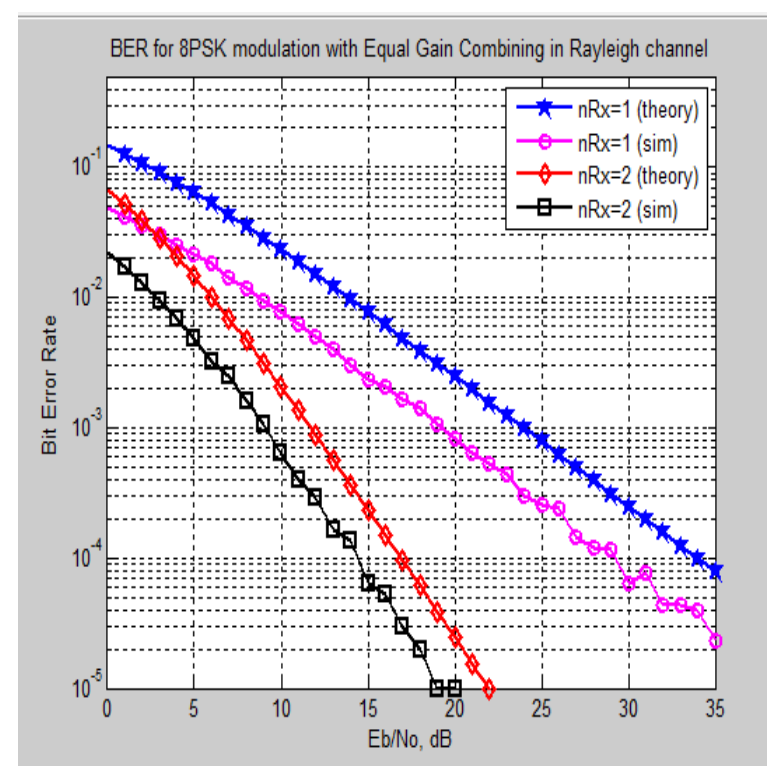

Fig 5: Bit Error Rate for 8-PSK using EGC 


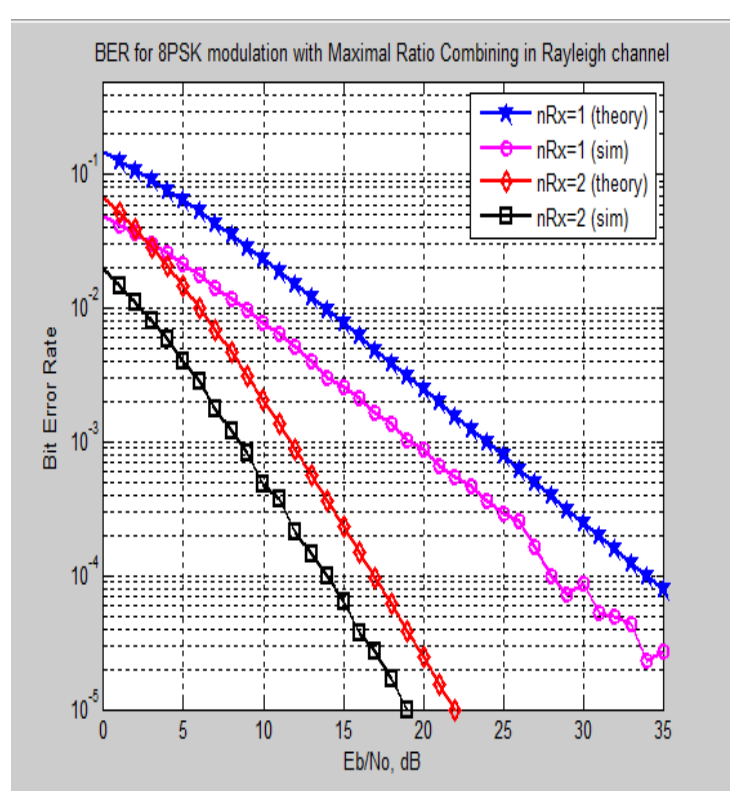

Fig 6: Bit Error Rate for 8-PSK using MRC

BER for 16PSK modulation with Equal Gain Combining in Rayleigh channel

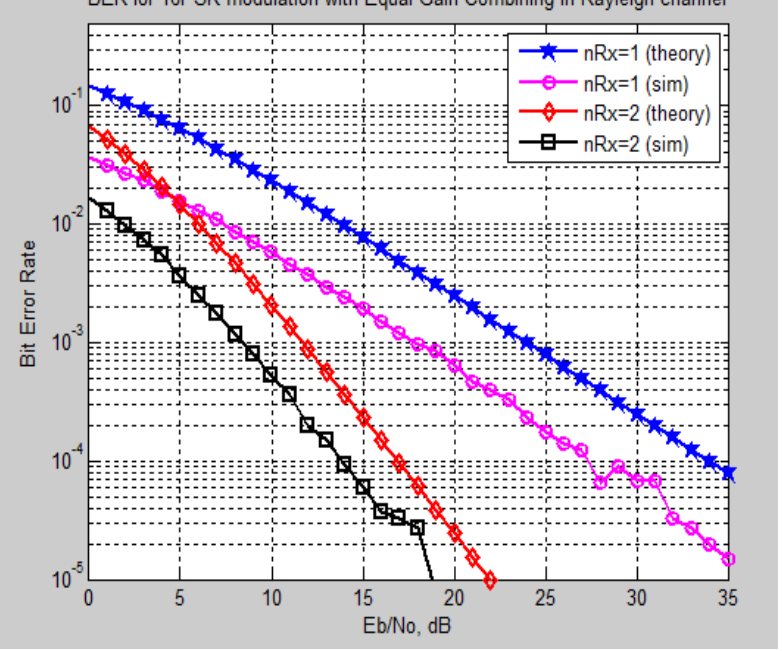

Fig 7: Bit Error Rate for 16-PSK using EGC

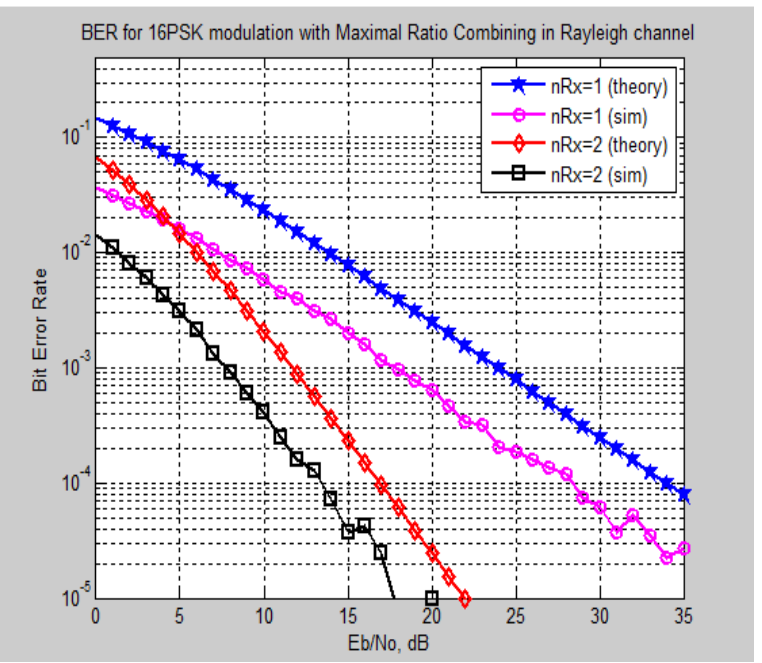

Fig 8: Bit Error Rate for 16-PSK using MRC

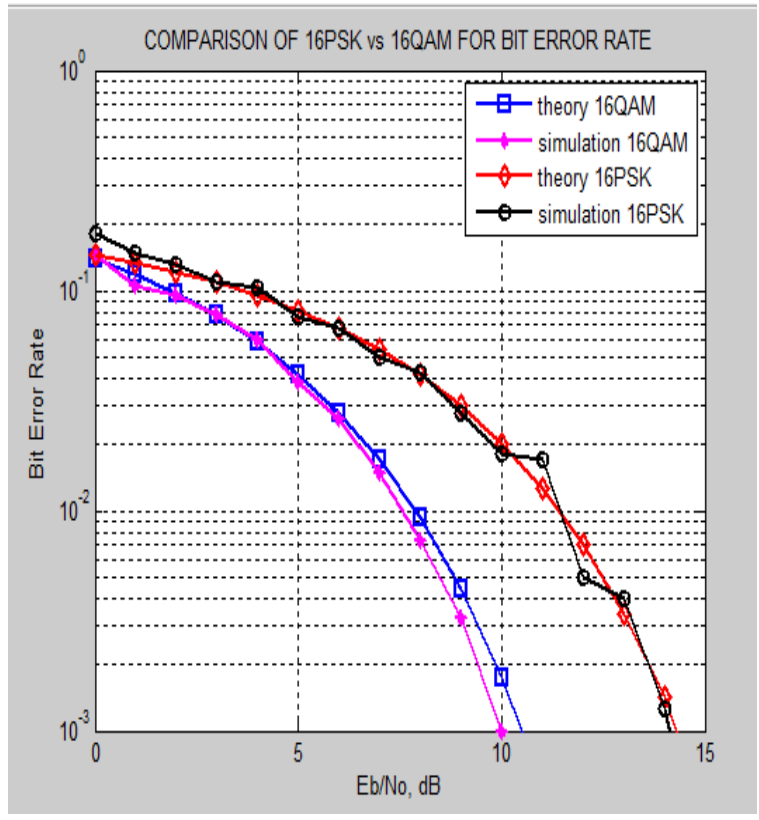

Fig 9: Comparison of 16 PSK and 16 QAM

It can be observed from Figure 5, Figure 6, Figure 7 and Figure 8 that the Bit Error Rate performance in Rayleigh Fading Channel is better for MRC as compare to EGC with increase in number of receive antennas. In case of $n R x=2$, both $M R C$ and EGC give better results, however, $n R x=1$ gives fluctuated results. This shows that with increse in number of antenna, BER is reduced and performance of the overall system is better for both diversity combining methods. Figure 9 shows the graph between the simulated and theoretical values of BER for 16 PSK and 16 QAM. It can be clearly seen from the graph that the bit error rate for 16 QAM is comparatively less than the bit error rate for 16 PSK. Therefore as the value of signal to noise ratio is increased the bit error rate is improved more in 16 QAM than 16 PSK. Therefore QAM techniques are more preferred over PSK techniques for all combining methods.

\section{CONCLUSION}

In this paper, SNR and BER for SIMO communication system are calculated with different modulation techniques like 8-PSK, 16-PSK and 16-QAM for MRC and EGC combining methods. As the number of antenna on receiver side is increased, the SNR is increased and BER is decreased. It is analyzed that when the values of SNR and BER are calculated for both combining methods, MRC gives the better result as compare to EGC. In this framework, QAM technique has shown better results than PSK for both combining methods. This type of scheme is high in demand because of their versatility features as high data rates, throughput, and different frequency of operability as per demand offered. Therefore, proposed algorithm is appropriate for fast moving vehicle as well as for fading environment conditions.

\section{REFERENCES}

[1] 1.M. K. Simon and M-S. Alouini, Digital Communication over Fading Channels. 1st ed., New York: Wiley, 2000.

[2] Wireless Communications Principles and Practice, Dr. Rappaport.

[3] . Dr. Narayan Mandayam, Rutgers University, Wireless Communication Technologies, lecture notes, Spring 2005.

[4] Fangming He, Hong Man and Wei Wang, "Maximal Ratio Diversity Combining Enhanced Security", IEEE 
Communications Letters, Vol-15, Issue: 5, pp-509 - 511, May 2011.

[5] Sheng-Chou Lin, "Performance analysis for optimum transmission and comparison with maximal ratio transmission for MIMO systems with co-channel interference", EURASIP Journal on Wireless Communications and Networking, 2011.

[6] B. Wang, "Accurate BER of transmitter antenna selection/receiver-MRC over arbitrarily correlated Nakagami fading channels," in Proc. IEEE ICASSP'06, Vol. 4, Toulouse, France, May 2006, pp. 753-756.

[7] David J. Love and Robert W.Heath, "Equal Gain Transmission in Multiple-Input Multiple-Output Wireless
Systems", IEEE TRANSACTIONS ON COMMUNICATIONS, 2003.

[8] Z. Wang and G. B. Giannakis, "A simple and general parameterization quantifying performance in fading channels," IEEE Trans. Commun. , Vol. 51, no. 8, pp. 1389-1398, Aug. 2003

[9] P. Gupta and P. R Kumar, " The capacity of wireless networks," IEEE Trans.Info. Theory, vol. 46, no. 2, pp. 388-404, Mar. 2002.

[10] Rizvi, Yilmaz, Janssen, Weber and louin, "Performance of Equal Gain Combining with Quantized Phases in Rayleigh Fading Channels", IEEE Transactions on Communications, Vol-59, Issue: 1 , pp-13 - 18, January, 2011. 